\title{
Prenatal Diagnosis of Congenital Dermal Sinus
}

\author{
Sharif Sakr, MD, MSc ${ }^{1}$ Yedathore Mohan, MD² Asif Malik, MD, FAAP ${ }^{3}$ Ghaus Malik, MD² \\ Bernard Gonik, MD ${ }^{1}$
}

\footnotetext{
${ }^{1}$ Department of Obstetrics and Gynecology, Wayne State University, Detroit, Michigan

2 Department of Neurosurgery, Henry Ford West Bloomfield Hospital, West Bloomfield, Michigan

${ }^{3}$ Department of Anesthesiology, Henry Ford West Bloomfield

Hospital, West Bloomfield, Michigan
}

\author{
Address for correspondence Bernard Gonik, MD, Department of \\ Obstetrics and Gynecology, Wayne State University School of \\ Medicine, 3990 John R Street, 7-Brush N, Box 163, Detroit, MI 48201 \\ (e-mail: bgonik@med.wayne.edu).
}

Am J Perinatol Rep 2015;5:e43-e45.

\begin{abstract}
Keywords

- fetal

- prenatal diagnosis

- congenital dermal sinus

- neural tube defect

Background Congenital dermal sinus (CDS) is an uncommon form of spinal dysraphism. Although postdelivery identification in the neonate is aided by several associated physical examination findings, establishing this diagnosis prenatally has proven to be elusive.

Case Report We present a case of CDS where the prenatal findings at 20 weeks gestation led to the diagnosis, which was confirmed postnatally. The associated protrusion of fibrotic membranes through the sinus tract helped in the identification of this lesion prenatally, but created confusion with a more common type of lesion, an open neural tube defect. This is the first case report in the literature describing prenatal diagnosis of fetal CDS.

Conclusion Prenatal diagnosis with postnatal confirmation of CDS leads to early intervention, better long-term outcomes, and lesser complications.
\end{abstract}

\section{Case Report}

A 35-year-old female patient, gravida 2, para 1, was referred for consultation following a routine office fetal anatomy scan at 20 weeks' gestation that suggested a neural tube defect (NTD). The patient had a normal first trimester aneuploidy screen and a second trimester maternal serum $\alpha$-fetoprotein (AFP) level of 0.71 multiples of median. With repeat ultrasound scanning, fetal biometric measurements were consistent with the patient's stated gestational age. The intracranial anatomy was normal, without any abnormalities in the posterior fossa and with normal cerebellar peduncles and normal cerebellar diameters. There was no evidence of ventriculomegaly or associated "lemon" or "banana" signs. In the lower lumbar region of the fetal spine, there appeared to be a thin, free-floating $11 \mathrm{~mm}$ finger-like sacculation projecting off the midline portion of one of the lower lumbosacral vertebrae (-Fig. 1A, B). Some images also suggested a small skin defect in that area. Genetic amniocentesis was performed and demonstrated a normal karyotype (except for a pericentric inversion of chromosome 9) with a normal amniotic fluid AFP and negative for acetyl cholinesterase. Fetal magnetic resonance imaging (MRI) confirmed the ultrasound findings of normal appearing brain, ventricles, and posterior fossa structures and absence of fetal spine masses, gross segmentation anomalies, obvious NTD or conus abnormality. The soft tissue protrusion in the lumbosacral region was seen, slightly eccentric to the left. The patient was counseled as to the suspected spinal dysraphism diagnosis, with this most likely representing a dermal sinus defect. The patient continued through the pregnancy without complications. She underwent delivery by elective repeat cesarean section at term of an appropriately grown female fetus, with good Apgar scores. At delivery, examination of the neonate was normal except for the identification of a $3 \mathrm{~cm}$ translucent skin appendage in the lumbar region (-Fig. 2). On day 2, the baby was evaluated by the neurosurgery service. No neurologic deficits were received

September 23, 2014 accepted after revision

December 4, 2014

published online

March 2, 2015
Dol http://dx.doi.org/ 10.1055/s-0034-1544108. ISSN 2157-6998.
Copyright (c) 2015 by Thieme Medical Publishers, Inc., 333 Seventh Avenue, New York, NY 10001, USA. Tel: +1(212) 584-4662.
License terms

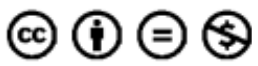



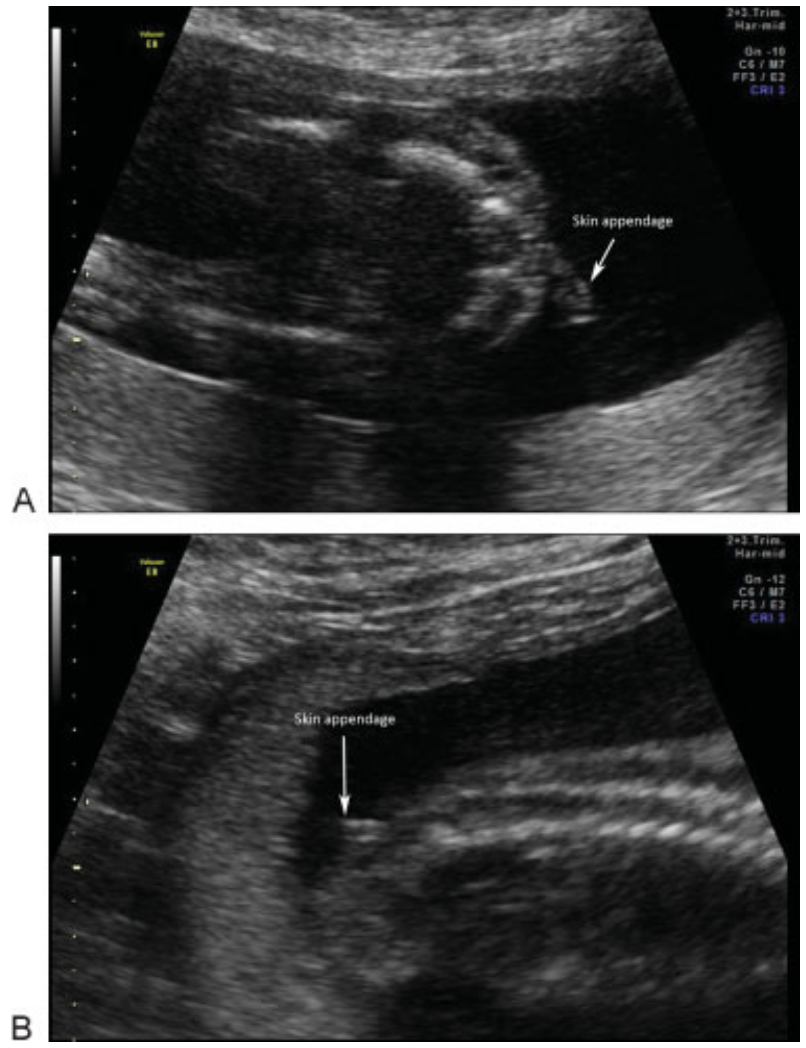

Fig. 1 A and B: Prenatal two-dimensional ultrasound showing a freefloating finger-like sacculation in the lumbar region.

identified. MRI of the spine confirmed the diagnosis of dorsal dermal sinus extending from dural sac, between L4 and L5 and superiorly to the $3 \mathrm{~cm}$ skin appendage at the L3 to L4 level (-Fig. 3). There was no tethering of the spinal cord and the vertebral bodies were noted to be intact. On the 8th day of life, surgery was performed, including excision of the dermal sinus tract and resection of the meningeal tenting at the level of the dural sac. The skin around the dermal sinus was excised then undermined and using the operating microscope, the sinus was dissected along its margins until the dural sac was reached (-Fig. 4). No leakage of cerebrospinal fluid was observed from the dural sac, nor was there any neural tissue

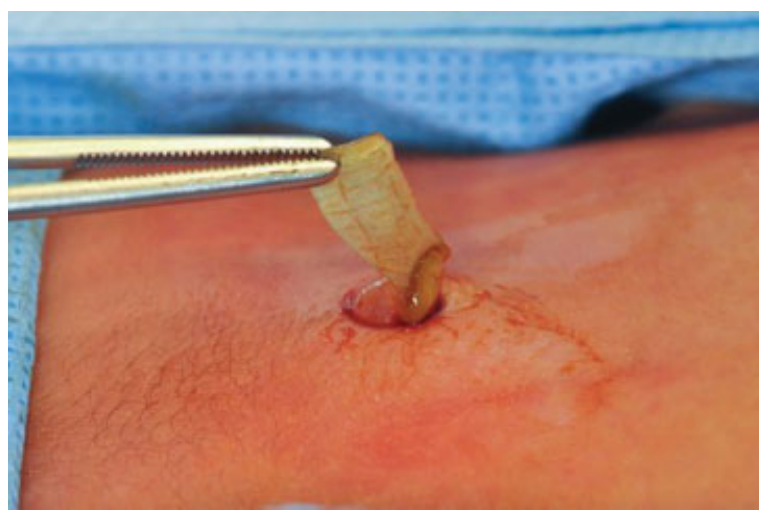

Fig. 2 Neonatal gross appearance of skin appendage.

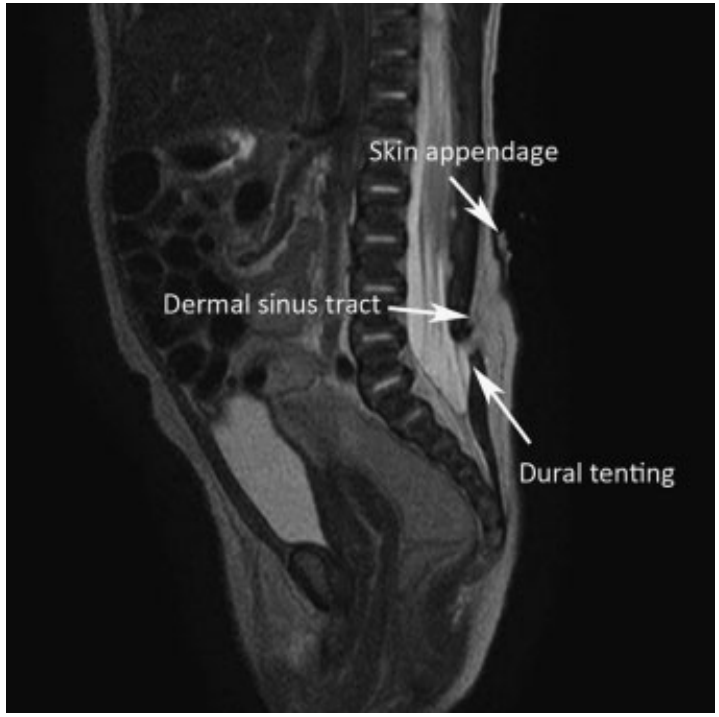

Fig. 3 Neonatal magnetic resonance imaging of the lumbar spine with radiologic diagnosis of dermal sinus tract, dural tenting, and skin appendage.

in the tract. The tract was closed and flaps were dissected to provide for defect closure. The diagnosis of lumbar dermal sinus tract with associated meningeal tenting was confirmed by the histopathologic examination. Postoperatively, the neonate recovered uneventfully.

\section{Discussion}

A congenital dermal sinus (CDS) is an uncommon form of spinal dysraphism resulting from nondisjunction. Disjunction normally takes place between 3 and 8 weeks of gestation. Embryologically, it entails the separation of the cutaneous ectoderm (future skin and dermal appendages) from the neuroectoderm (future spinal cord). ${ }^{1}$ This separation allows for the insertion of mesoderm between these two primordial layers to form vertebrae and muscles thus anatomically separating the skin from the spinal canal. Failure of such a process results in the persistence of a tract between the skin and neural structures.

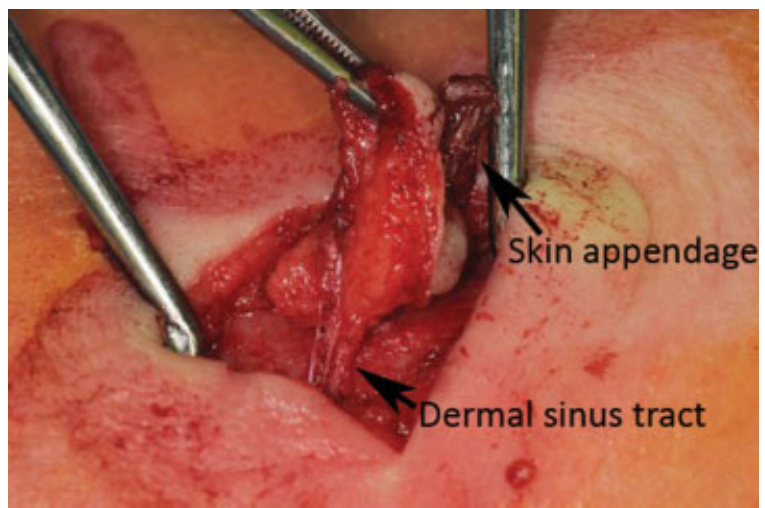

Fig. 4 Surgical view of the skin appendage and the dermal sinus dissected under the skin. 
CDS tracts are relatively rare, with an incidence rate of approximately 1 in 2,500 live births. The majority occur in the lumbar and sacral regions. ${ }^{2,3}$ Common presentations after birth include skin findings, infection or neurological deficit. Skin findings include skin tags, abnormal pigmentation, hypertrichosis, subcutaneous lipomas, and angioma. CDS can also be seen in association with other pathology, such as inclusion tumors, split cord malformations, and tethered cords. ${ }^{1}$ Two hundred cases of occult spinal dysraphism were reviewed by Tavafoghi et al, noting a high association, more than $50 \%$, with skin lesions such as skin dimples or sinuses, subcutaneous tumors, hemangiomas, hypertrichosis, and hyperpigmentation. ${ }^{4}$ Treatment is surgical excision, with successful results in more than $90 \%$ of the cases. ${ }^{5}$

Using PubMed search criteria of congenital dermal sinus and prenatal diagnosis, we were unable to identify any previous reports in the literature of this diagnosis being established during the antenatal period. In one case report, the identification of a fluid-filled lumbar cyst during a prenatal ultrasound examination led to the suspected diagnosis of meningocele, which postnatally proved to be a CDS. ${ }^{6}$ In another case report, an occipital tumor without intracranial communication was found on ultrasonography and with MRI at 21 weeks' gestation. However, the precise diagnosis of CDS in association with a hemangioma was only established after delivery with surgical resection and with histologic confirmation at 6 months of age. ${ }^{7}$ In the present case, the patient was originally referred to our center with the presumptive diagnosis of a NTD because of the ultrasonographically visualized lumbar sacculation in the fetus. Given the lack of other features of NTD, in particular the absence of bony abnormalities below this lesion, we were led to consider other less common forms of spinal dysraphism. This was key, in which the prognostic implications of broadening the differential diagnosis were significant. Despite the need for surgical intervention, as stated above, long-term outcomes are generally more favorable with CDS than with typical NTD lesions.

As a part of the prenatal differential diagnosis, we considered that the ultrasound findings may have represented a vestigial human tail. This appendage appears to be the result of a disturbance in the normal embryologic regression of the human tail bud. ${ }^{8}$ Vestigial tails typically have adipose tissue, connective tissue, striated muscle, blood vessels, and nerve tissue as components. Pseudo tails have also been described, and these have been defined to have only bony or nervous system elements. ${ }^{9}$ Both these structures may be isolated or in association with other anatomic abnormalities, such as meningoceles, spina bifida occulta, lipomas, and tethered cord. ${ }^{10}$ Vestigial tails have been identified with prenatal ultrasonography and have a variable prognosis depending on associated anomalies. ${ }^{11-14}$

Despite the generally more favorable prognosis of CDS, a careful neonatal neurologic assessment is in order, including imaging of the spinal canal and cord. We were fortunate not to find any evidence of split or tethered spinal cord in our neonate, although both have been reported with spinal dysraphism. ${ }^{5,15}$ CDS was found to be often associated with vertebral abnormalities: bifid lamina, fused vertebrae, and scoliosis were noted in 17, 3, and 3 of the 28 patients, respectively. ${ }^{5}$ In 2003, Ackerman et al highlighted how early postnatal diagnosis has a great impact on improving the outcome and avoiding long-term complications. ${ }^{5}$ In their study, fewer patients (50\%) with CDS diagnosed before 1 year, versus $90 \%$ of the patients diagnosed after 1 year of age, had long-term neurologic deficits, such as sensory changes, motor weakness, reflex and gait changes, and decreased sphincter function. Delayed diagnosis and management also increases the risks of infection (abscess/ meningitis), increased mass size, and difficulty in resection and repair.

\section{References}

1 French BN. The embryology of spinal dysraphism. Clin Neurosurg 1983;30:295-340

2 McINTOSH R, Merritt KK, Richards MR, Samuels MH, Bellows MT. The incidence of congenital malformations: a study of 5,964 pregnancies. Pediatrics 1954;14(5):505-522

3 French BN. Midline fusion defects and defects of formation. In: JR Y, ed. Neurological Surgery. Philadelphia: WB Saunders Company; 1990:1081-235

4 Tavafoghi V, Ghandchi A, Hambrick GW Jr, Udverhelyi GB. Cutaneous signs of spinal dysraphism. Report of a patient with a taillike lipoma and review of 200 cases in the literature. Arch Dermatol 1978;114(4):573-577

5 Ackerman LL, Menezes AH. Spinal congenital dermal sinuses: a 30-year experience. Pediatrics 2003;112(3 Pt 1):641-647

6 Hamill N, Grant JA, Myers SA. Congenital dermal sinus. J Ultrasound Med 2008;27(5):799-802

7 Viskova H, Calda P, Zizka Z, Vaneckova M, Hoza D, Zuntova A. Prenatal diagnosis of occipital dermal sinus associated with hemangioma using ultrasound and MRI. Case report. Fetal Diagn Ther 2006;21(2):232-234

8 Fallon JF, Simandl BK. Evidence of a role for cell death in the disappearance of the embryonic human tail. Am J Anat 1978; 152(1):111-129

9 Dao AH, Netsky MG. Human tails and pseudotails. Hum Pathol 1984;15(5):449-453

10 Lu FL, Wang PJ, Teng RJ, Yau KI. The human tail. Pediatr Neurol 1998;19(3):230-233

11 Abbott JF, Davis GH, Endicott B, Pfleghaar K, Wapner RJ. Prenatal diagnosis of vestigial tail. J Ultrasound Med 1992;11(1):53-55

12 Guven MA, Uzel M, Ceylaner S, Coskun A, Ceylaner G, Gungoren A. A prenatally diagnosed case of sirenomelia with polydactyly and vestigial tail. Genet Couns 2008;19(4):419-424

13 Harirah H, Hsu C, Bahado-Singh R, Copel JA. Human caudal appendage diagnosed prenatally with ultrasound. Obstet Gynecol 2000;95(6 Pt 2):1038

14 Dobson LJ, Barnewolt CE, Morash D, Connolly SA, Estroff JA. Human fetal sacrococcygeal extension or 'tail' in the second trimester: prenatal diagnosis, associated findings, and clinical outcome. Prenat Diagn 2013;33(2):134-140

15 Pang D, Dias MS, Ahab-Barmada M. Split cord malformation: Part I: a unified theory of embryogenesis for double spinal cord malformations. Neurosurgery 1992;31(3):451-480 